\title{
Naringenin Enhances Anti-Proliferation Effect of FMSP On K562 Human Chronic Myelogenous Leukemia Cells Via Targeting Calmodulin Signaling Pathway
}

Sadegh Rajabi ( $\sim$ sadegh.rajabi2017@gmail.com )

Shaheed Beheshti University of Medical Sciences https://orcid.org/0000-0003-0177-7922

Shokoofe Noori

Shahid Beheshti University of Medical Sciences School of Medicine

Mohammad Reza Ashrafi

Kerman University of Medical Sciences

Mahsa Azami Movahed

Shahid Beheshti University of Medical Sciences School of Pharmacy

Shabnam Farzaneh

Shahid Beheshti University of Medical Sciences School of Pharmacy

Afshin Zarghi

Shahid Beheshti University of Medical Sciences School of Pharmacy

\section{Research Article}

Keywords: Chronic myelogenous leukemia, FMSP, Naringenin, CAMP, PKA, PDE-1

Posted Date: August 4th, 2021

DOl: https://doi.org/10.21203/rs.3.rs-735694/v1

License: (c) (i) This work is licensed under a Creative Commons Attribution 4.0 International License.

Read Full License 


\section{Abstract}

Background: FMSP is a synthesized ferrocene derivative which possesses strong anti-proliferative and apoptosis inducing characteristics on tumor cells. Naringenin as a polyphenolic flavonoid is also able to reduce cell viability in tumors.

Methods: Cell viability and proliferation of cancer cells after treatment with these agents were determined with MTT assay. To predict the possible interaction between calmodulin (CaM) and FMSP and naringenin, docking stuies were performed. By using fluorescence emission spectra, effects of FMSP and naringenin on CaM structure and activity were studied. CaM-dependent activation of phosphodiesterase 1 (PDE1) by FMSP in relation to naringenin and their combination were compared. Effects of these compounds on PDE1 inhibition, cAMP accumulation, and cAMP-dependent protein kinase A (PKA) activation were assayed.

Results: Our results showed that combination of FMSP and naringenin had more inhibitory effects on CaM structure than FMSP and naringenin alone. Results of docking analyses also confirmed efficient intraction of the two compunds with hydrophobic pocket of calmodulin active site. Kinetic analyses of these agents interaction with CaM showed FMSP and naringenin both competitively inhibited PDE1 activation without changing Vmax parameter. FMSP and naringenin synergistically increased $\mathrm{Km}$ values in a higher level compared to FMSP or naringenin alone. Combination of these two agents also had more cytotoxic effects on K562 cells than FMSP alone.

Conclusions: It was shown that mechanism of K562 cell proliferation inhibition by these compounds is based on CaM and consequent PDE inhibition followed by intracellular cAMP level elevation and increased PKA activity in a dose-dependent manner.

\section{Introduction}

Chronic myelogenous leukemia $(\mathrm{CML})$ is a neoplastic hematopoietic disease that more commonly occurs in men (50-60 years-old) and is presented by an uncontrollable proliferation of mature granulocytes [1]. CML has been estimated to cause 8990 new cases and 1140 deaths in the United States during 2019 [2]. Different treatment options are available for CML patients but several adverse side effects may occur due to this therapeutics [3]. Therefore, finding novel and effective therapeutic agents with few or no potential adverse effects is necessary. Discovery of various molecular pathways involved in the cancer has led to the development novel molecular targeted treatments that suppress cancer growth [4]. These targeted therapies mainly act by blocking specific molecules required for cell proliferation and tumorigenesis. Specific targeting of key molecules in cancer cells renders these new therapies a higher efficacy and fewer adverse effects in comparison to other conventional therapies [5]. Currently, tyrosine kinase inhibitors (TKIs) have reported as the successful and efficient molecular targeted therapeutics for the treatment of patients with CML, however, some patients show resistance to TKI therapy and need alternative therapeutic approaches to tackle the disease [6]. 
Cyclic adenosine monophosphate (cAMP) is an intracellular messenger molecule that is able to stimulate cell proliferation in various cell types while inhibit this process in some others. This molecule exerts its effects by the activation of cAMP-dependent protein kinase A (PKA) [7]. Study of cancer cells also has shown that an elevated level of intracellular cAMP plays a pro-apoptotic role within these cells [8]. The intracellular concentration of CAMP is mainly under the control of two enzymes including adenylyl cyclases and cyclic nucleotide phosphodiesterases (PDEs), which are implicated in the synthesis and degradation CAMP, respectively [9]. PDE1, as one of the isoforms of this enzyme, has a binding site for Ca2+/calmodulin (CaM) in its structure by which PDE1 is activated to degrade CAMP in a CaM-dependent manner [10]. It has been revealed that inhibition of PDE1 could cause apoptosis induction in cancer cells and hence this enzyme could be of great importance as a therapeutic strategy to trigger apoptosis of cancer cells [11]. Furthermore, calmodulin itself has been proven to stimulate apoptosis and cell cycle blockage both in-vitro and in-vivo[12].

Previous studies have indicated that plant-derived phytochemicals have significant ant- cancer activities both alone or in combination with synthetic compounds $[13,14]$. Results of our previous investigation showed that 1-ferrocenyl-3-(4-methylsulfonylphenyl)propen-1-one (FMSP, Fig. 1), which was synthetized by our team, as a ferrocene derivative robustly decreased viability of tumor cells in-vitro [15]. Naringenin, as a natural polyphenolic flavonoid, has anti-proliferative and apoptosis-inducing properties in many cancer cell types [16-18]. In this study, the effects of FMSP and naringenin on CaM, PDE1, and the cAMP/PKA signaling pathway were investigated in order to reveal their mechanism of action in cell growth inhibition.

\section{Material And Methods}

\section{Preparation of FMSP}

According to our previously reported protocol, acetyl ferrocene reacted with 4(methylsulfonyl)benzaldehyde in sodium hydroxide and ethanol to yield 1-ferrocenyl-3-(4methylsulfonylphenyl)propen-1-one (FMSP) [19]. The synthesized compound was well characterized by elemental analysis, multinuclear $\left({ }^{1} \mathrm{H}\right) \mathrm{NMR}$, and spectroscopic methods such as IR and Mass.

\section{Molecular modeling and docking studies}

Docking studies were performed using AutoDock software version 4.0 to search for favorable binding configurations between the small flexible ligands and the rigid protein to predict their interactions. These studies were performed based on the high-resolution crystal structure of calmodulin receptor (PDB code: 1 QIW) retrieved from RCSB Protein Data Bank. The co-crystallized ligand (DPD) and water molecules were removed from the protein, Kollman charges were added, nonpolar hydrogens were merged, and AutoDock 4 atom type was assigned to achieve the PDBQT format of the protein. The ligand structures (Naringenin and FMSP) were minimized using HyperChem8.0 ( $\mathrm{MM}^{+}$method) and then converted to PDBQT file format with AutoDock tools. A docking grid box was built with $(30 \times 30 \times 30)$ surround the co- 
crystallized ligand. The Lamarckian genetic search algorithm was employed, and the docking run was set to 100. Protein residues with atoms greater than $6.0 \AA$ from the docking box were removed for efficiency. The quality of the docked structures was evaluated by measuring the intermolecular energy of the ligand-enzyme assembly.

\section{Determination of kinetic parameters}

We used $\Delta \mathrm{G}(\mathrm{H} 2 \mathrm{O})$ as an appropriate parameter to calculate macromolecular stability of CaM protein in the presence of FMSP and naringenin. The free energy difference $(\Delta G)$ between the native and denatured forms of CaM was calculated by below formula [20]:

$\Delta \mathrm{G}=-\mathrm{RT} \operatorname{Ln}[\mathrm{Fd} /(1-\mathrm{Fd})]$

$F_{d}=\left(Y_{n}-Y_{o b s}\right) /\left(Y_{n-} Y_{d}\right)(1)$

$\operatorname{Ln}\left[\left(Y_{n}-Y_{o b s}\right) /\left(Y_{o b s}-Y_{d}\right)\right]=-R T L n K$

In this formula, $\mathrm{R}$ stands for gas constant, $\mathrm{T}$ represents the absolute temperature, $\mathrm{Y}_{\text {obs }}$ indicates fluorescence emission intensity of CaM at different concentrations of FMSP and naringenin, and $Y_{n}$ and $Y_{d}$ stand for values of native and denatured states, respectively. The changes in $\Delta G$ values were plotted against FMSP and naringenin concentrations, which showed a linear relation based on the following equation [20]:

$\Delta \mathrm{G}=\Delta \mathrm{G}\left(\mathrm{H}_{2} \mathrm{O}\right)-\mathrm{m}[\mathrm{D}](2)$

In the above mentioned formula, $\Delta \mathrm{G}\left(\mathrm{H}_{2} \mathrm{O}\right)$ stands for $\Delta \mathrm{G}$ at zero concentration of ligand, $\mathrm{m}$ is the constant for the dependence of $\Delta G$ on ligand concentration, and $[D]$ is the ligand concentration.

Lineweaver-burk plots were created by kinetic analysis of CaM-dependent PDE inhibition in the presence of FMSP and naringenin. Thereafter, in the presence and absence of these two inhibitors, $\mathrm{K}_{\mathrm{m}}$ values calculated from these plots. According to the plots, maximum velocity (Vmax) of the activated PDE1 in the presence and absence of inhibitors is the point at which the lines crossed the y-axis.

\section{Cell culture}

The K562 human erythroleukemic cell line was provided by Pasteur Institute, Tehran, Iran. Subsequently, the cancer cells were cultured in RPMI 1640 culture medium (Thermo Fisher Scientific, USA), which were supplemented with 10\%FBS (Gibco, Germany), 100 unit/ml penicillin/100 mg/ ml streptomycin (Thermo Fisher Scientific, USA), and 1\% L-glutamine (Gibco, Germany). The cultured cells were incubated in $5 \%$ $\mathrm{CO} 2$ at $37^{\circ} \mathrm{C}$ conditions.

\section{Cell viability analysis}

Cell viability estimation was carried out using 3-[4, 5- methylthiazol-2-yl]-2, 5-diphenyl-tetrazolium bromide (MTT) assay. In brief, the cultured cells were trypsinized and collected to be seeded in 96-well tissue 
culture plates (5000 cells/well) overnight. Then, the cells were treated with various concentrations of FMSP $(0-5 \mu \mathrm{M})$ and naringenin $(50 \mu \mathrm{M})$ for $48 \mathrm{~h}$. Subsequently, a solution containing $10 \% \mathrm{MTT}$ was prepared using non-complete culture medium and was added to the well for $4 \mathrm{~h}$. After 4-h incubation, MTT-contained medium was replaced by $100 \mu \mathrm{L}$ Dimethyl sulfoxide (DMSO) (Sigma-Aldrich Company, USA) to solve the produced MTT crystals. Finally, the absorbance of the solution was read by using an ELISA reader at $570 \mathrm{~nm}$ and the cell viabilities were determined as the percentage of untreated control cells.

\section{Quantitative determination of cAMP concentration}

The concentration of cAMP molecules within the cells was measured by an enzyme immunoassay kit (Stratagene, La Jolla, CA). Briefly, after treating the K562 cells with the mentioned concentrations of FMSP and naringenin in treatment group and culture medium in controls, about $2 \times 10^{5}$ cells were collected and centrifuged at $1200 \mathrm{rpm}$ and $4^{\circ} \mathrm{C}$ for $5 \mathrm{~min}$. Cell pellets resuspended by the lysis buffer provided in the kit. According to the kit protocols, cAMP concentration was instantly measured in lysed cells. This method was principally based on the competition between unlabeled cAMP and stable quantity of peroxidase-labeled cAMP for the limited binding sites on an antibody against cAMP molecule.

\section{Measurement of PKA activity}

In this present study, a colorimetric assay kit (BioVision, USA) was used for determination of PKA enzyme activity. Briefly, the treated K562 cells were washed with Phosphate-buffered saline (PBS) buffer ( $\mathrm{pH}=$ 7.4) twice, and the resuspended by lysis buffer (50mMTris- $\mathrm{HCl}, 2.5 \mathrm{mMEDTA}, 1 \mathrm{mMMgCl} 2,10 \mathrm{mMNaF}$, $10 \%$ glycerol, $\mathrm{pH} 7.2$ ) and sonicated followed by centrifugation at $1200 \mathrm{rpm}$ and $4^{\circ} \mathrm{C}$ for $5 \mathrm{~min}$. Finally, the activity of PKA enzyme was measured according to the instructions that were provided by the manufacturer.

\section{Effects of PKA blocking on cell growth inhibition}

Rp-cAMP (Adenosine 3,5-cyclic monophosphorothioate triethyammonium salt hydrate) was used as a specific inhibitor of PKA (Biolog Life Science Institute, Bremen, Germany). This molecular inhibitor was then diluted by using RPMI culture medium to prepare $100 \mu \mathrm{M}$ Rp-cAMP. Briefly, the cultured cells were first incubated with Rp-cAMP $(100 \mu \mathrm{M})$ for 20 min and then treated with FMSP $(0-5 \mu \mathrm{M})$ and its combination with naringenin $(50 \mu \mathrm{M})$.

\section{Statistical analysis}

To assess the normality of data and homogeneity of variances, by K-S and Levene's statistical tests were used, respectively. Then, one-way analysis of variance (ANOVA) or Mann-Whitney nonparametric tests were applied to analyze the results of the study. The statistical significance of the obtained results was considered at $p<0.05$ level between the treated and control groups. Data were analyzed by using SPSS software version 22 , and results were indicated as the mean \pm standard error (mean \pm SE).

\section{Results}




\section{Molecular modeling and docking studies}

The calmodulin receptor pocket was found to be composed of two subunits ( $A$ and $B$ ) occupied by the cocrystallized inhibitor (DPD). The docking analysis was performed on subunit B of calmodulin.

As shown in Figs. 2A, B, and C, docking results showed that naringenin and FMSP structures exactly fit into the same hydrophobic pocket of calmodulin active site containing Phe92, Met124, Met144, and Met 145 as is occupied by the co-crystallized ligand. As specified in the figures, in the docking of naringenin, $\mathrm{OH}$ groups form hydrogen binds to Glu11 side chain carboxylic acid and lle100 oxygen atom. The FMSP is perfectly superimposed on the naringenin structure. This molecule participates in hydrophobic interaction with hydrophobic residues of Phe12, Phe92, Met144 and Met145 and also $\pi-\pi$ interactions with aromatic rings of Phe12 and Phe92.

\section{Interaction of FMSP and naringenin with calmodulin}

Alteration of fluorescence emission spectra of CaM illustrated the interaction of FMSP and naringenin ligands with $\mathrm{CaM}$ as well as drug-induced conformational change in this protein. These data represents inhibitory effects of these two compounds on CaM (Fig. 3A).

\section{Effect of FMSP and naringenin on the activity of PDE-1}

The activity of PDE-1 enzyme was measured by increasing concentrations of FMSP, naringenin, and their combination, respectively. This method is based on the hydrolysis of cAMP to AMP, which was quantified by using spectrophotometric technique. Finally, the activity of PDE-1 was calculated and plotted versus different concentrations of FMSP and naringenin (Fig. 3B).

\section{Kinetic analysis of PDE1 activity and inhibition of PDE1 by FMSP and naringenin}

Kinetic analysis of PDE-1 enzyme was calculated in the presence and absence of FMSP and naringenin. In the absence of both compounds, the Km value of PDE1 was $0.05 \pm 0.001 \mu \mathrm{M}$; but adding FMSP and naringenin alone and their combination together increased the $\mathrm{Km}$ values to $1,0.1$ and $3 \mu \mathrm{M}$, respectively. These data indicated that the presence of FMSP and naringenin increases the $\mathrm{Km}$ values in a concentration-dependent manner. Moreover, FMSP and naringenin increased $\mathrm{Km}$ values with no apparent impact on $\mathrm{V}_{\max }$ values. The efficacy order of the combination of FMSP and naringenin, FMSP, and naringenin in increasing $\mathrm{Km}$ values is as follow: FMSP + Naringenin $>$ FMSP $>$ Naringenin. According to the lineweaver-burk plots, either FMSP or naringenin or in combination together competitively inhibit PDE1 enzyme (Fig. 4A). To show the effectiveness of an inhibitor Ki constant is used. This parameter indicates the affinity of the inhibitor to a specific enzyme. We calculated the Ki values of our enzymeinhibitor reactions by Dickson plot (Figs. 4B,C, and D). The determined Ki constants for FMSP and naringenin and their combination were 5,10 , and 0.5 respectively.

\section{The effect of FMSP and naringenin on calmodulin stability}


The stability of CaM protein was estimated in the presence of FMSP, Naringenin and the combination of these two agents separately. The CaM free energy changes $(\Delta G)$ for complexes of CaM with FMSP and naringenin alone and their combination are shown in Fig. 5. According to the linear plots, the estimated $\Delta \mathrm{G}(\mathrm{H} 2 \mathrm{O})$ in the presence of FMSP, naringenin, and the combination of FMSP and naringenin was 1.8, 2.4 , and $0.2 \mathrm{kcal} \mathrm{mol}^{-1}$, while this value was $7.1 \mathrm{kcal} \mathrm{mol}^{-1}$ in absence of these two compounds (Table 1).

Table 1

Calmodulin stability parameters in the presence of Naringenin and FMSP

\begin{tabular}{|c|c|c|c|c|c|}
\hline Ligand & Concentration $(\mu \mathrm{M})$ & $\begin{array}{l}\Delta \mathrm{G}(\mathrm{H} 2 \mathrm{O}) \\
(\mathrm{kCal} / \mathrm{mol})\end{array}$ & $\begin{array}{l}\mathrm{m}(\mathrm{kCal} / \mathrm{mol} / \\
\mu \mathrm{M})\end{array}$ & $\begin{array}{l}\mathrm{D} 1 / 2 \\
(\mu \mathrm{M})\end{array}$ & R2 \\
\hline Calmodulin & 5 & 7.1 & 0.101 & 69.7 & 0.99 \\
\hline Naringenin & 50 & 2.4 & 0.042 & 55.3 & 0.99 \\
\hline FMSP & 5 & 1.8 & 0.035 & 51.4 & 0.99 \\
\hline $\begin{array}{l}\text { FMSP + } \\
\text { Naringenin }\end{array}$ & $50+5$ & 0.2 & 0.0151 & 13.17 & 0.99 \\
\hline
\end{tabular}

To examine the effect of FMSP alone and its combination with naringenin on the proliferation of K562 cells, different doses of FMSP $(0-5 \mu \mathrm{M})$ with a single dose of naringenin $(50 \mu \mathrm{M})$ were studied by using MTT assay. Results showed that FMSP alone significantly $(p<0.05)$ reduced the proliferation of these cancer cells in doses more than $2 \mu \mathrm{M}$. Moreover, the combination of FMSP with naringenin with the mentioned doses also meaningfully $(p<0.05)$ diminished the viability of K562 cells in comparison to untreated control cells. Interestingly, the combination of FMSP and naringenin was more effective than FMSP alone in hindering proliferation of human K562 cells (Fig. 6A).

\section{The effect of FMSP alone and its combination with naringenin on intracellular levels of cAMP and PKA activity in $\mathrm{K} 562$ cells}

Enzyme immunoassay method was used to evaluate the effects of FMSP $(0-5 \mu \mathrm{M})$ alone and its combination with naringenin $(50 \mu \mathrm{M})$ on cAMP concentration and PKA activity within K562 cell line. Treatment of K562 cells with FMSP and naringenin considerably augmented the levels of intracellular cAMP in these cancer cells (Fig. 6B and C). Furthermore, the exposure of K562 cells to both FMSP alone and its combination with naringenin showed a significant increase in PKA activity compared with control group (Fig. 6D). Interestingly, the activity PKA and the levels of cAMP in combination treatment group were non-significantly higher than the FMSP-treated group. 


\section{Activation of PKA by cAMP is required for cell growth inhibition by FMSP alone and its combination with naringenin}

To evaluate whether the inhibitory effects of FMSP alone and its combination with naringenin on the viability of K562 cells is mediated by PDE inhibition and the consequent elevation of cAMP and PKA activation, the cancer cells were pretreated with Rp-cAMP as a specific inhibitor of PKA for 20min. Then, the cells were treated with FMSP $(0-5 \mu \mathrm{M})$ alone and its combination with naringenin $(50 \mu \mathrm{M})$ and MTT assay was used to measure the viability of the cells. As indicated in the Fig. 6B, pretreatment of K562 cell line with the mentioned doses of the inhibitor and treatment of them with two drugs significantly decreased the viability of these cancer cells in comparison to controls. This suggests that the cAMPdependent activation of PKA is necessary to inhibit the cell proliferation due to FMSP and naringenin treatments.

\section{Discussion}

The CaM protein has been reported to regulate different cellular processes including proliferation, apoptosis, autophagy, migration, metastasis, metabolic homeostasis, and expression of various genes [21]. As mentioned before, CaM binds PDE1 to activate this enzyme to degrade intracellular cAMP molecule [10]. PDE1 is involved in the regulation of proliferation and apoptosis in cancer cells [22]. Consequently, emerging importance of CaM antagonists in the treatment of cancer patients has been shown many years ago [23]. Additionally, PDE inhibitors have been developed for the treatment of different diseases [24]. Furthermore, it has been revealed that PDE1 inhibitors are able to kill tumor cells selectively without affecting normal cells [25]. Based on our previous study, FMSP hindered the expression of stemness markers and decreased viability of breast cancer cells by reducing proliferation and inducing apoptosis in these cells [15]. Naringenin has been reported to exert anti-cancer effects on different cells via proliferation inhibition and apoptosis induction [26, 27].

In this study, the first step was carried out to examine the effects of FMSP and naringenin on CaM structure. Our results showed that the combination of FMSP and naringenin efficiently induced conformational alterations on CaM protein and increased its fluorescence emission. As shown in Fig. 3A, inhibitory activity of these two compounds and their combination against CaM is as follows: FMSP + Naringenin > FMSP > Naringenin. The second step of this study was done to assess the impacts of CaM activation on PDE1 activity in the presence of FMSP and naringenin, and FMSP + naringenin compared with untreated control. It was revealed that the combination of FMSP and naringenin more significantly inhibited PDE1 activity than FMSP and naringenin alone. As indicated in Fig. 4A, the Ki constant for FMSP + naringenin was considerably higher than those of FMSP and naringenin alone. Combination of the two compounds synergistically incremented Km value of PDE1 without any changes in Vmax parameter. These results indicate that these agents competitively inhibit PDE activity. 
The data of $\Delta \mathrm{G}(\mathrm{H} 2 \mathrm{O})$ values presented in Fig. 3 are consistent with findings that were mentioned above. The decrease in CaM stability could be attributed to the reduction of $\triangle \mathrm{G}(\mathrm{H} 2 \mathrm{O})$ values due to its interaction with the mentioned FMSP and naringenin. Combination of FMSP and naringenin more remarkably decreased $\triangle \mathrm{G}(\mathrm{H} 2 \mathrm{O})$ values than FMSP and naringenin alone, indicating that the combined drugs more efficiently decreased CaM stability when compared with single compounds.

In the third step, the effects of FMSP and naringenin on the viability of K562 cell line were evaluated invitro. The results showed that the combination of FMSP and naringenin more significantly declined the proliferation of K562 cells in comparison to FMSP and naringenin alone (Fig. 6A). We also unraveled that the combination of FMSP and naringenin had more significant effect on the induction of PKA activity and elevation of cAMP levels compared with FMSP alone (Fig. 6B and C).

To evaluate the dependence of the inhibition of cell growth by FMSP and naringenin on PKA activation, effects of these agents on PKA activity were examined. It was revealed that either the combination of FMSP and naringenin or FMSP alone enhanced the activity of PKA (Fig. 6D). In the last step, to understand whether PKA has a role in growth-inhibitory activity of these compounds, K562 cells were preincubated with PKA inhibitor (Rp-CAMP) and then treated with FMSP + naringenin and FMSP alone. Our findings revealed that in the presence of Rp-cAMP, inhibitory effects of FMSP + naringenin and FMSP alone on K562 cells was mediated by the accumulation of cAMP and activation of PKA.

\section{Conclusion}

Taken together, this study indicated that FMSP is a more potent antagonist of CaM than naringenin. Naringenin could enhance the antagonizing effects of FMSP on CaM protein. By antagonizing CaM, these compounds inhibit PDE1 and consequently increase CAMP levels and PKA activity. Combination of FMSP and naringenin and FMSP alone, reduce K562 cells proliferation in a dose-dependent manner possibly via cAMP/PKA pathway by CaM and PDE1 inhibition. Since both FMSP and naringenin are able to hamper viability of cancer cells, they can be promising anti-proliferative and also pro-apoptotic agents and this necessitate the requirement to investigate their potential anticancer affects in the future researches.

\section{Declarations}

Acknowledgments: The authors thank the Vice Chancellor for Research of Shahid Beheshti University of Medical Sciences.

Funding: No funding resources were received.

Conflicts of interest: There is no conflict of ontereest to declare.

Availability of data and material: Our data is available upon any request from the corresponding author. 
Author Contributions: Shokoofe Noori and Afshin Zarghi designed the experiment and research methodology. Mahsa Azami Movahed and Shabnam Farzaneh performed the experiments, while Sadegh Rajabi and Mohammad Reza Ashrafi were involved in the data analysis and manuscript write-up.

Consent to participate: Not applicabale.

Consent for publication: All authors approved the publication.

\section{References}

1. Sampaio MM, Santos MLC, Marques HS, Gonçalves VLS, Araújo GRL, Lopes LW et al (2021) Chronic myeloid leukemia-from the Philadelphia chromosome to specific target drugs: A literature review. World J Clin Oncol 12:69-94. https://doi.org/10.5306/wjco.v12.i2.69

2. Sarno F, Pepe G, Termolino P, Carafa V, Massaro C, Merciai F et al (2020) Trifolium Repens Blocks Proliferation in Chronic Myelogenous Leukemia via the BCR-ABL/STAT 5 Pathway. Cells 9:379. https://doi.org/10.3390/cells9020379

3. Jabbour E, Kantarjian H (2020) Chronic myeloid leukemia: 2020 update on diagnosis, therapy and monitoring. Am J Hematol 95:691-709. https://doi.org/10.1002/ajh.25792

4. Nouri Z, Fakhri S, Nouri K, Wallace CE, Farzaei MH, Bishayee A (2020) Targeting Multiple Signaling Pathways in Cancer: The Rutin Therapeutic Approach. Cancers (Basel) 12:2276. https://doi.org/10.3390/cancers12082276

5. Seebacher NA, Stacy AE, Porter GM, Merlot AM (2019) Clinical development of targeted and immune based anti-cancer therapies. J Exp Clin Cancer Res 38:156. https://doi.org/10.1186/s13046-0191094-2

6. Soverini S, Mancini M, Bavaro L, Cavo M, Martinelli G (2018) Chronic myeloid leukemia: the paradigm of targeting oncogenic tyrosine kinase signaling and counteracting resistance for successful cancer therapy. Mol Cancer 17:49. https://doi.org/10.1186/s12943-018-0780-6

7. Roberts MJ, May LT, Keen AC, Liu B, Lam T, Charlton SJ et al (2021) Inhibition of the Proliferation of Human Lung Fibroblasts by Prostacyclin Receptor Agonists is Linked to a Sustained cAMP Signal in the Nucleus. Front Pharmacol 12:669227. https://doi.org/10.3389/fphar.2021.669227

8. Insel PA, Wilderman A, Zhang L, Keshwani MM, Zambon AC (2014) Cyclic AMP/PKA-promoted apoptosis: insights from studies of S49 lymphoma cells. Horm Metab Res 46:854-862. https://doi.org/10.1055/s-0034-1384519

9. Epstein PM (2017) Different phosphodiesterases (PDEs) regulate distinct phosphoproteomes during cAMP signaling. Proc Natl Acad Sci U S A 114:7741-7743.

https://doi.org/10.1073/pnas.1709073114

10. Omori K, Kotera J (2007) Overview of PDEs and their regulation. Circ Res 100:309-327. https://doi.org/10.1161/01.RES.0000256354.95791.f1 
11. Shimizu K, Murata T, Watanabe Y, Sato C, Morita H, Tagawa T (2009) Characterization of phosphodiesterase 1 in human malignant melanoma cell lines. Anticancer Res 29:1119-1122

12. Yokokura S, Yurimoto S, Matsuoka A, Imataki O, Dobashi H, Bandoh S et al (2014) Calmodulin antagonists induce cell cycle arrest and apoptosis in vitro and inhibit tumor growth in vivo in human multiple myeloma. BMC Cancer 14:882. https://doi.org/10.1186/1471-2407-14-882

13. Noori S, Rajabi S, Tavirani MR, Shokri B, Zarghi A (2021) Anti-Breast Cancer Activities of KetoprofenRGD Conjugate by Targeting Breast Cancer Stem-Like Cells and Parental Cells. Anticancer Agents Med Chem 21:1027-1036. https://doi.org/10.2174/1871520620666200908105416

14. Rajabi S, Shojaee M, Malmir A, Rezaei Tavirani M, Noori S (2020) Anti-Breast Cancer Activities of 8Hydroxydaidzein by Targeting Breast Cancer Stem-Like Cells. J Pharm Pharm Sci 23:47-57. https://doi.org/10.18433/jpps30981

15. Nourbakhsh M, Farzaneh S, Taghikhani A, Zarghi A, Noori S (2020) The Effect of a Newly Synthesized Ferrocene Derivative against MCF-7 Breast Cancer Cells and Spheroid Stem Cells through ROS Production and Inhibition of JAK2/STAT3 Signaling Pathway. Anticancer Agents Med Chem 20:875-886. https://doi.org/10.2174/1871520620666200101151743

16. Lim W, Park S, Bazer FW, Song G (2017) Naringenin-Induced Apoptotic Cell Death in Prostate Cancer Cells Is Mediated via the PI3K/AKT and MAPK Signaling Pathways. J Cell Biochem 118:1118-1131. https://doi.org/10.1002/jcb.25729

17. Zhou J, Xia L, Zhang Y (2019) Naringin inhibits thyroid cancer cell proliferation and induces cell apoptosis through repressing PI3K/AKT pathway. Pathol Res Pract 215:152707. https://doi.org/10.1016/j.prp.2019.152707

18. Bao L, Liu F, Guo HB, Li Y, Tan BB, Zhang WX et al (2016) Naringenin inhibits proliferation, migration, and invasion as well as induces apoptosis of gastric cancer SGC7901 cell line by downregulation of AKT pathway. Tumour Biol 37:11365-11374. https://doi.org/10.1007/s13277-016-5013-2

19. Farzaneh S, Zeinalzadeh E, Daraei B, Shahhosseini S, Zarghi A (2018) New Ferrocene Compounds as Selective Cyclooxygenase (COX-2) Inhibitors: Design, Synthesis, Cytotoxicity and Enzyme-inhibitory Activity. Anticancer Agents Med Chem 18:295-301. https://doi.org/10.2174/1871520617666171003145533

20. Pace CN (1990) Measuring and increasing protein stability. Trends Biotech 8:93-98. https://doi.org/https://doi.org/10.1016/0167-7799(90)90146-0

21. Villalobo A, Berchtold MW (2020) The Role of Calmodulin in Tumor Cell Migration, Invasiveness, and Metastasis. Int J Mol Sci 21:765. https://doi.org/10.3390/ijms21030765

22. Noori S, Hassan ZM (2014) Tehranolide inhibits cell proliferation via calmodulin inhibition, PDE, and PKA activation. Tumour Biol 35:257-264. https://doi.org/10.1007/s13277-013-1031-5

23. Hait WN, Morris S, Lazo JS, Figlin RJ, Durivage HJ, White K et al (1989) Phase I trial of combined therapy with bleomycin and the calmodulin antagonist, trifluoperazine. Cancer Chemo Pharmacol 23:358-362. https://doi.org/10.1007/bf00435836 
24. Keravis T, Lugnier C (2012) Cyclic nucleotide phosphodiesterase (PDE) isozymes as targets of the intracellular signalling network: benefits of PDE inhibitors in various diseases and perspectives for future therapeutic developments. Br J Pharmacol 165:1288-1305. https://doi.org/10.1111/j.14765381.2011.01729.x

25. Peng T, Gong J, Jin Y, Zhou Y, Tong R, Wei X et al (2018) Inhibitors of phosphodiesterase as cancer therapeutics. Eur J Med Chem 150:742-756. https://doi.org/10.1016/j.ejmech.2018.03.046

26. Zhao Z, Jin G, Ge Y, Guo Z (2019) Naringenin inhibits migration of breast cancer cells via inflammatory and apoptosis cell signaling pathways. Inflammopharmacology 27:1021-1036. https://doi.org/10.1007/s10787-018-00556-3

27. Park HJ, Choi YJ, Lee JH, Nam MJ (2017) Naringenin causes ASK1-induced apoptosis via reactive oxygen species in human pancreatic cancer cells. Food Chem Toxicol 99:1-8. https://doi.org/10.1016/j.fct.2016.11.008

\section{Figures}

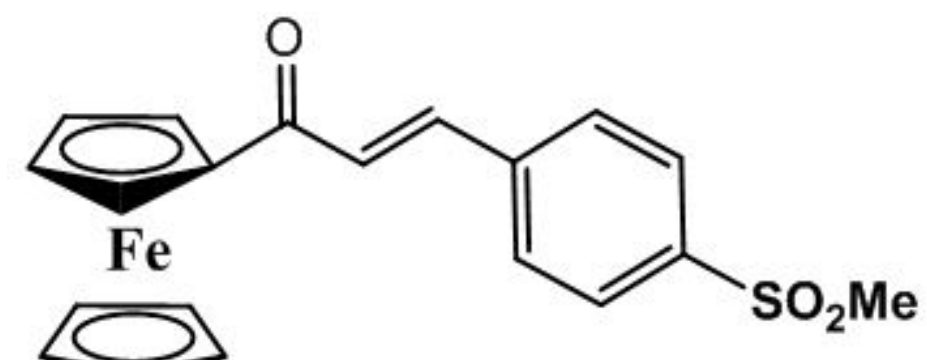

Figure 1

Structure of FMSP 

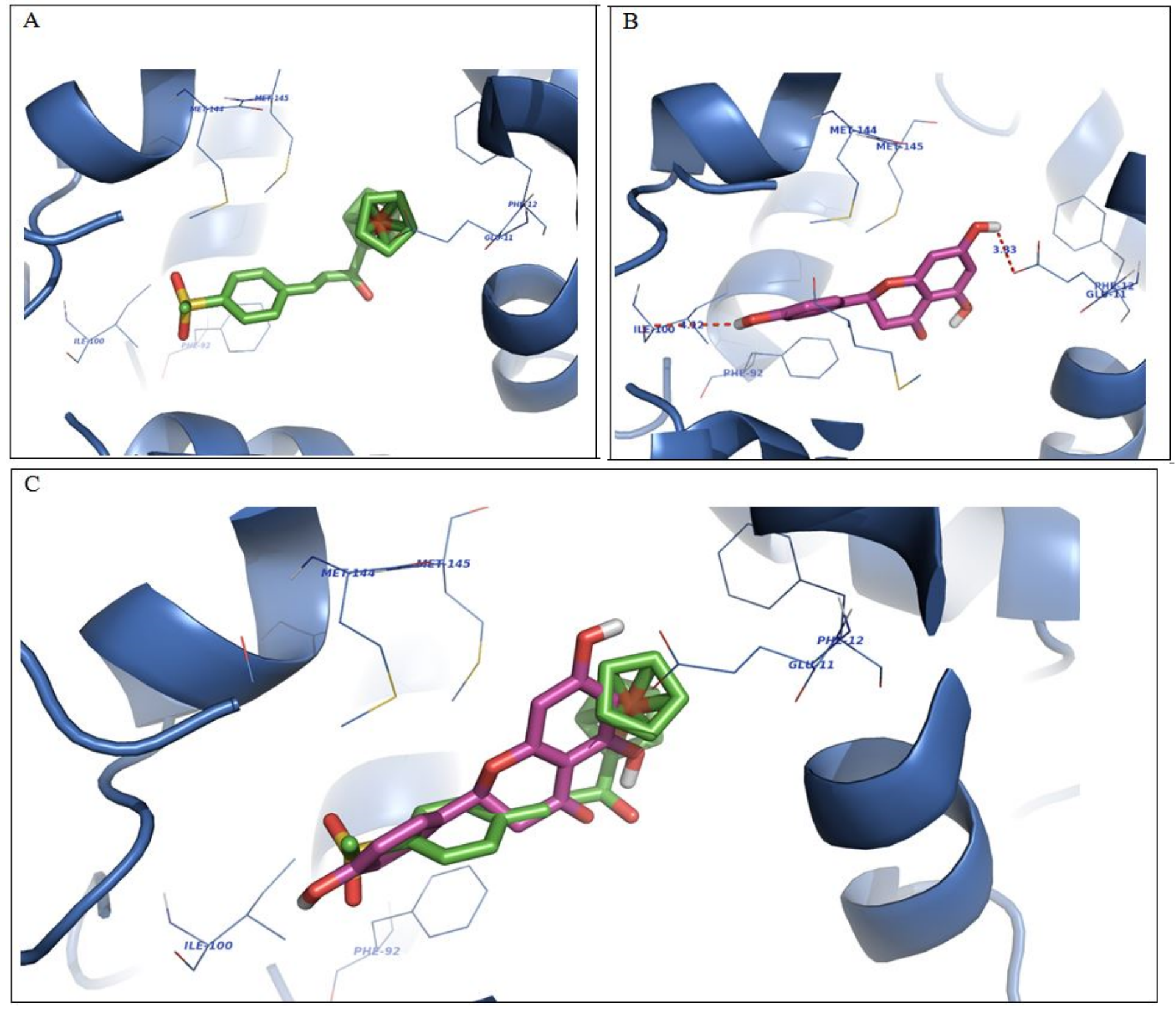

Figure 2

Results of ducking analysis of FMSP (A), naringenin (B) and superimposition of FMSP on naringenin (C) in calmodulin active site 

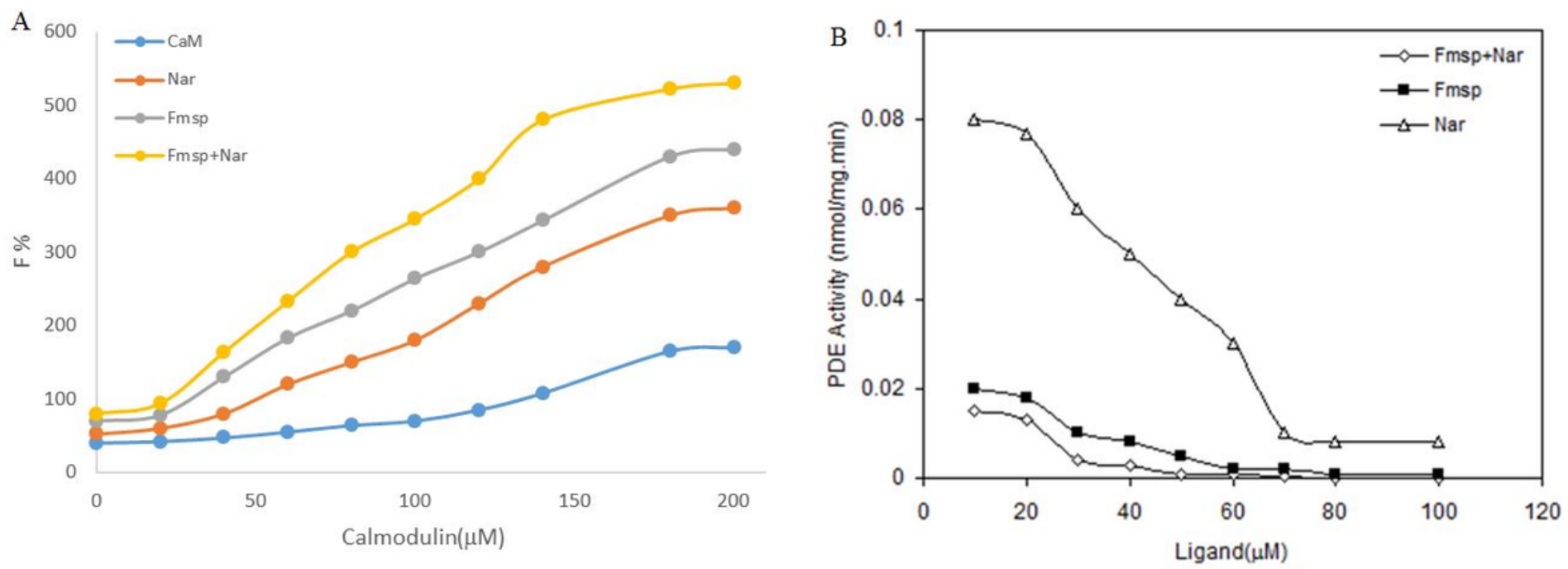

Figure 3

(A) Calmodulin (CaM) fluorescence spectra at $0.043 \mathrm{mg} / \mathrm{ml}$ concentration in $40 \mathrm{mM}$ Tris- $\mathrm{HCl}$ buffer, $\mathrm{pH}$ 7.4 at $25^{\circ} \mathrm{C}$. Elevation of calmodulin concentration in the free form and in the presence of $10 \mu \mathrm{M} \mathrm{FMSP}$, naringenin and their combination caused an increase in fluorescence emission of CaM. (B) Calmodulindependent activity of PDE1 against different concentrations of FMSP, naringenin, and their combination 


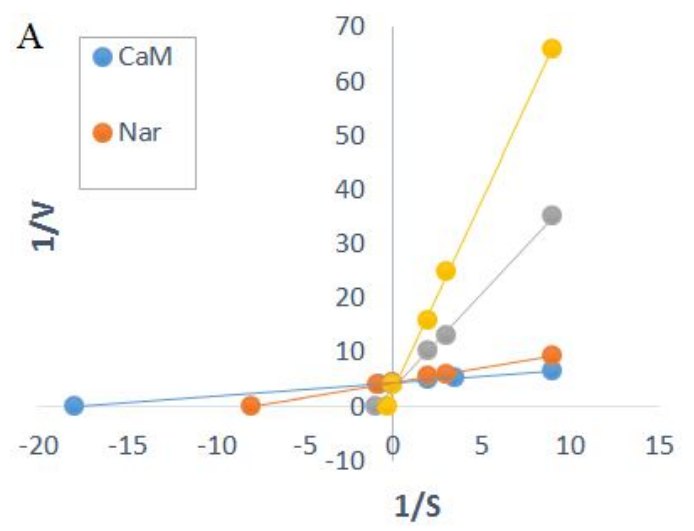

$\mathrm{C}$

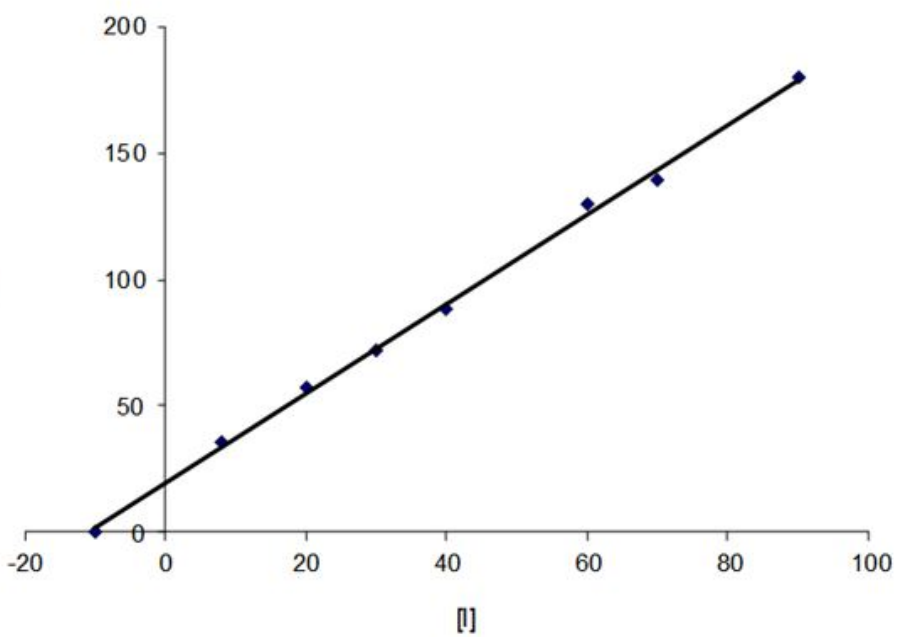

Fmsp

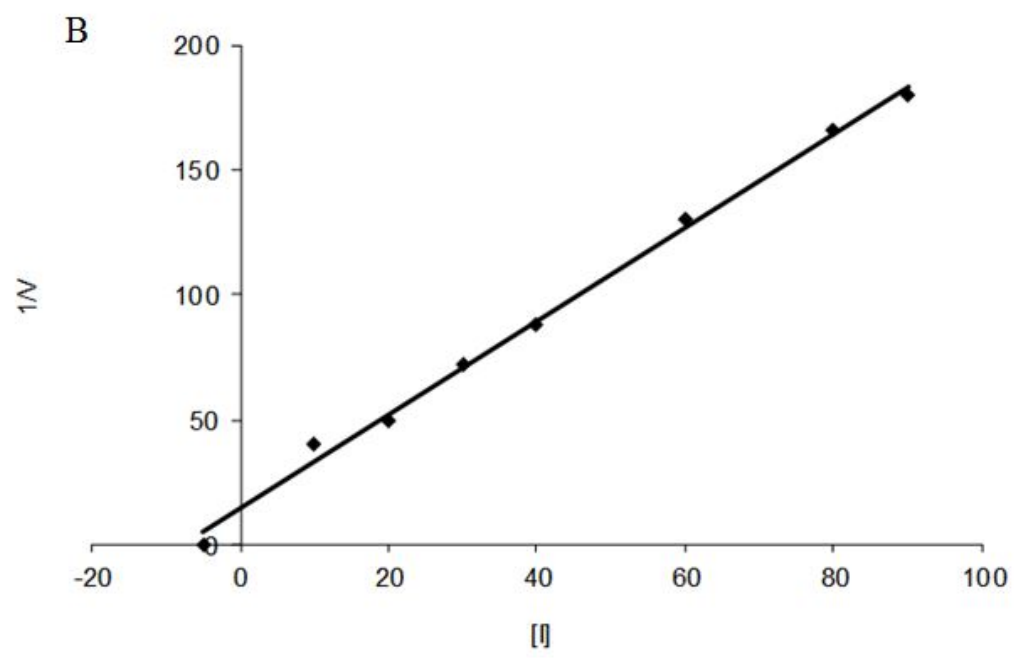

Fmsp+Nar

$\mathrm{D}$

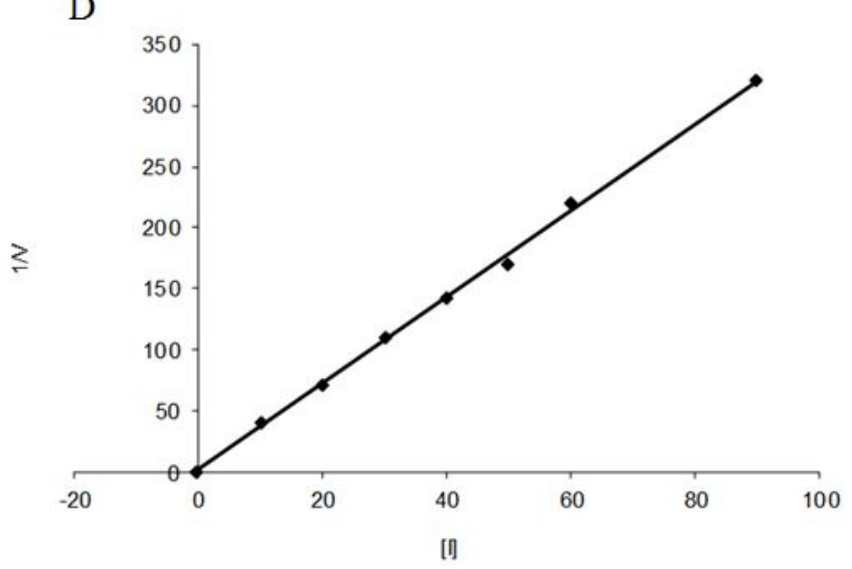

\section{Figure 4}

(A) Lineweaver-burk plot for the kinetic analysis of PDE1 activity and the mode of inhibition of the enzyme by FMSP and naringenin. Inhibition was performed with $5 \mu \mathrm{M} \mathrm{FMSP}$ and $50 \mu \mathrm{M}$ naringenin and their combination in different concentrations of cAMP (calmodulin concentration in all the reactions was constant, $100 \mu \mathrm{M})$. (B), (C) and (D) Dixon plots: Inhibition was performed with varying concentrations of both compounds and their combination 


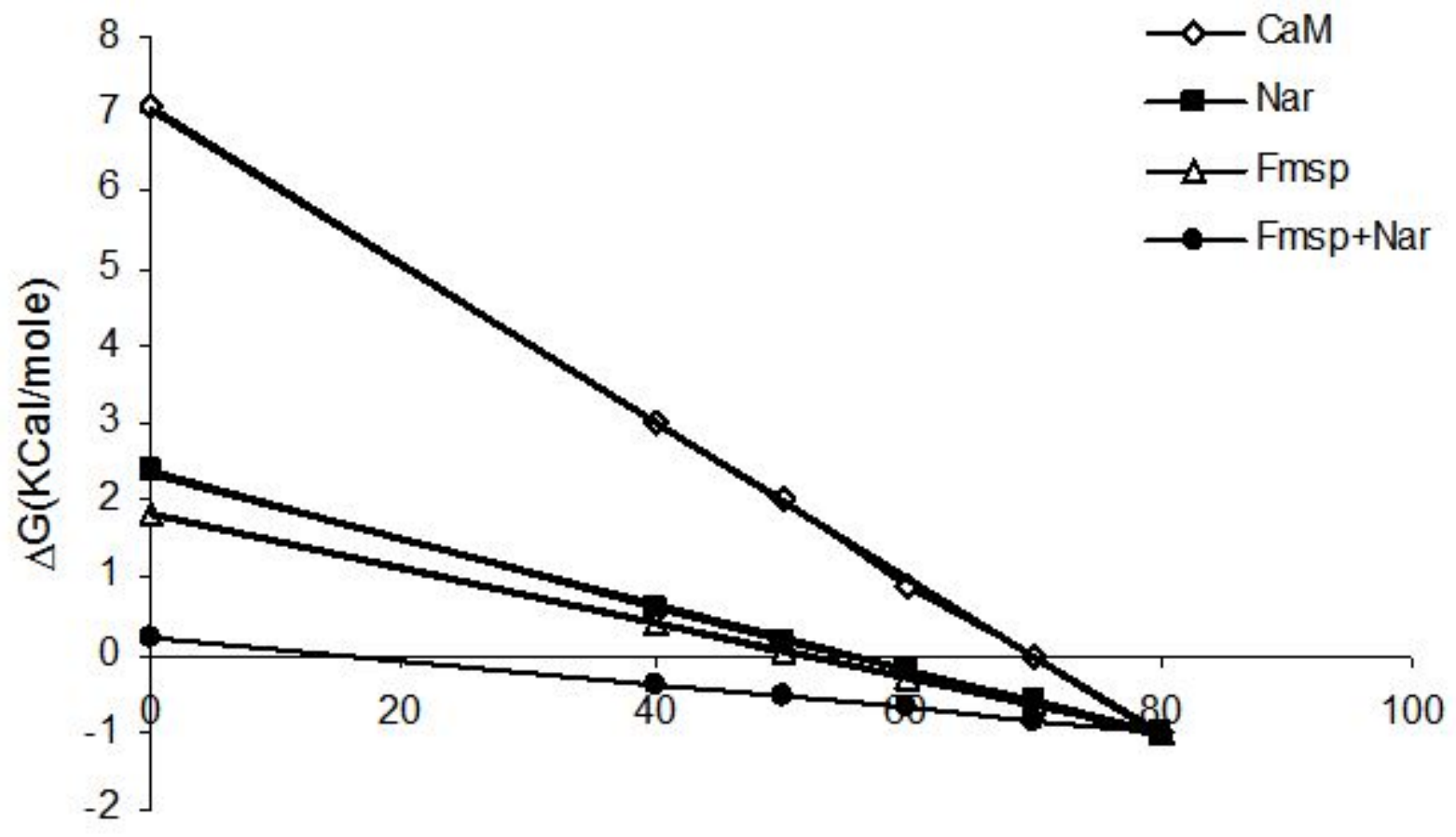

Ligand( $\mu \mathrm{M})$

\section{Figure 5}

The changes in the free energy $(\Delta G)$ of calmodulin against different concentrations of FMSP, naringenin and their combination 

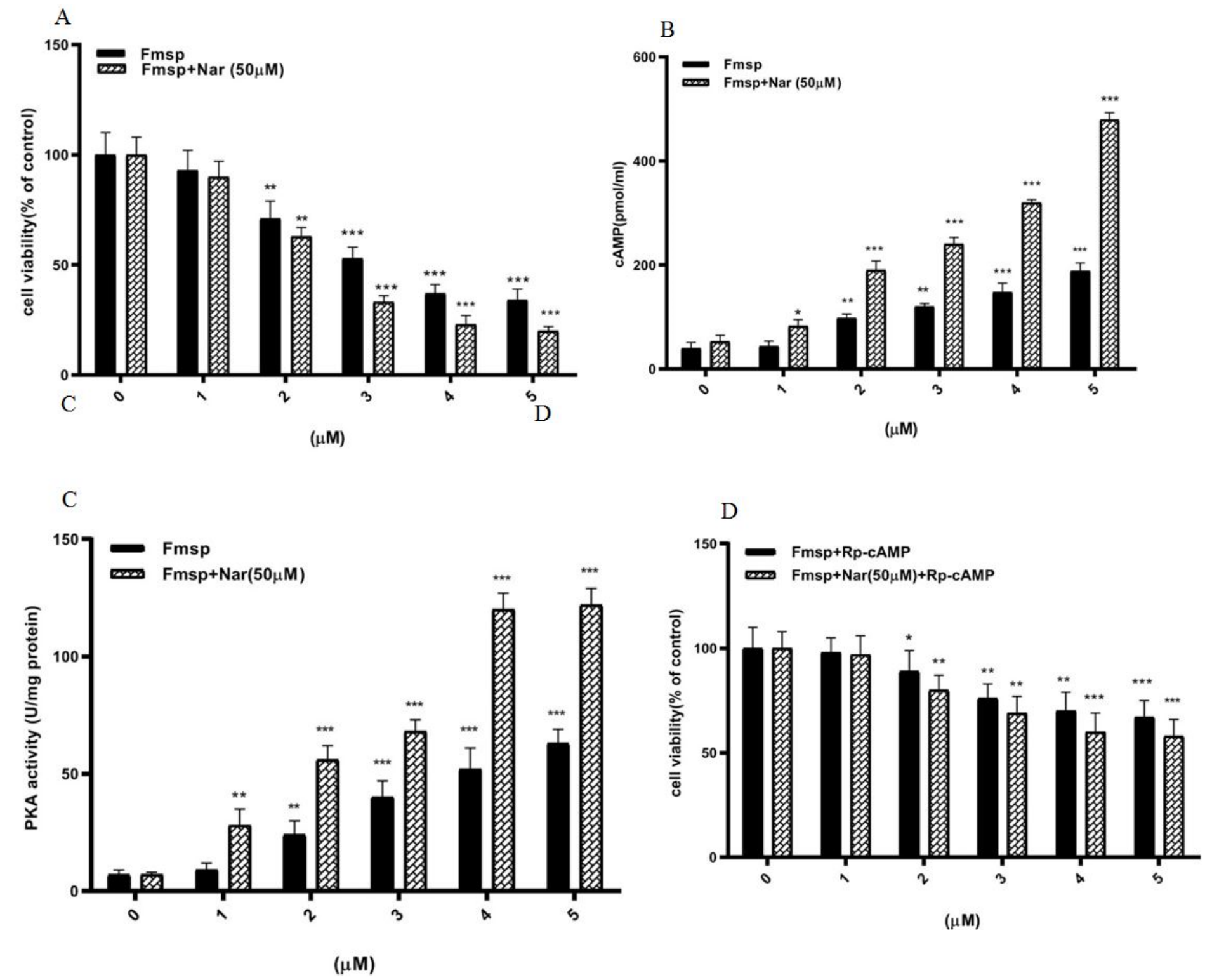

Figure 6

(A) Cytotoxicity impacts of FMSP and its combination with naringenin on K562 cell line. Effects of increasing concentrations of FMSP and its combination with naringenin on the viability of K562 cells were achieved by using MTT assay. Bars indicate the mean \pm SEM of the treatments that were carried out in triplicate. P-values less than 0.05 were indicative of significant differences compared with control group. (B) Intracellular levels of cAMP in K562 cells which were treated with different concentrations of FMSP and its combination with naringenin. (C) Determination of PKA activity in K562 cells by colorimetric assay kit. (D) Effect of the pretreatment of K562 cells with Rp-cAMP, as a specific inhibitor of PKA, in the presence of FMSP alone and its combination with naringenin 